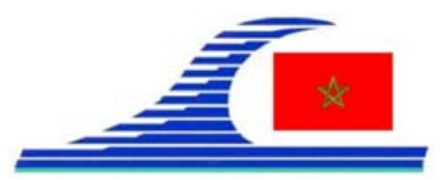

\title{
Aspects maritimes du dessalement d'eau de mer
}

\section{Jean BOUGIS ${ }^{1}$}

1. Ingénieur Conseil, 32 Chemin du Moulin, 06650 Opio, France. jean.bougis@wanadoo.fr

\section{Résumé :}

Depuis une trentaine d'années, la production d'eau douce par le dessalement d'eau de mer se développe rapidement pour différents usages : eau potable, usages domestiques et industriels, irrigation. Après un bref aperçu historique et le rappel des différents procédés industriels disponibles, les différents aspects maritimes sont abordés : modes de prise d'eau indirects et directs, rejet des saumures, contraintes environnementales, tenue à la mer des ouvrages, critères de choix physiques et économiques.

\section{Mots-clés :}

Dessalement d'eau de mer - Puits côtier - Puits maritime - Tranchée drainante - Drain par forage dirigé - Champ drainant - Prise d'eau directe - Rejet des saumures

\begin{abstract}
:
Since around thirty years, the production of fresh water from the desalination of sea water quickly develops for various uses: drinking water, domestic and industrial use, irrigation. After a brief historical reminder and an overview of various available industrials process, the various maritime aspects are approached: indirect or direct sea water intake facilities, brines discharge, environmental requirements, marine structures sea keeping, physical and economic criteria of choice.
\end{abstract}

\section{Keywords:}

Sea water desalination - Coastal well - Beach well - Subsurface flow drains - Horizontal directional drains - Seabed intake facility - Direct seawater intake - Brine discharge

\section{Aperçu historique}

Le dessalement (désalinisation ou dessalage) consiste à produire de l'eau douce à partir d'une eau salée ou saumâtre, généralement par extraction de l'eau douce et plus rarement par extraction du sel.

Les premières unités de dessalement d'eau de mer sont apparues avec les premiers bateaux à vapeur. L'usage du dessalement d'eau de mer pour la production d'eau douce (eau potable, usages domestiques et industriels, irrigation) s'est développé dans la seconde moitié du XX ${ }^{\text {ème }}$, essentiellement depuis le premier choc pétrolier de 1973. Jusque dans les années 1960, la distillation à faisceaux noyés était le seul procédé industriel disponible. La température de chauffe très élevée, nécessaire à l'obtention d’un rendement acceptable, engendrait de fréquents arrêts pour détartrer le bouilleur. Des 
La connaissance de la Mer :

un vecteur du développement durable en Méditerranée

procédés de distillation plus performants sont apparus à partir des années 1960 : FLASH, compression de vapeur, etc. A la même époque sont apparues les premières unités utilisant des procédés membranaires. Les progrès accomplis par le procédé d'osmose inverse (qualité des membranes, récupération d'énergie, baisse des coûts, ...) lui ont permis de s'imposer pour les petites et moyennes unités et de rivaliser avec les procédés de distillation pour les grosses unités.

\section{Procédés de dessalement d'eau de mer}

Parmi les nombreux procédés de dessalement, cinq seulement sont utilisés à l'échelle industrielle ; trois procédés de distillation et deux procédés membranaires (MAUREL, 2001) :

a) distillation à multiples effets (MED : Multi Effect Distillation),

b) distillation par détentes successives (MSF : Multi Stage FLASH),

c) distillation à compression de vapeur (VCD : Vapor Compression Distillation),

d) électrodialyse (ED : Electro Dialysis),

e) osmose inverse (OI ou RO : Reverse Osmosis).

Schématiquement, une installation de dessalement se compose de quatre unités :

a) la prise d'eau de mer brute et le rejet à la mer du concentrât ou saumure,

b) le prétraitement, particulièrement important pour les procédés membranaires : élimination des matières en suspension, des matières organiques, des "poisons des membranes", des éléments entartrant,

c) le dessalement proprement, c'est-à-dire la séparation du perméat et du concentrât,

d) le post-traitement pour rendre l'eau douce conforme aux normes selon les usages.

e) Les prétraitements doivent donc être plus ou moins poussés en fonction de la qualité de l'eau de mer brute disponible et du procédé de dessalement adopté.

\section{Différents types de prises d'eau de mer}

Il existe trois grandes familles de méthodes pour prélever de l'eau de mer brute. Elles présentent des sous-familles qui se déclinent en variantes selon des critères secondaires (particularités des sites, procédés technologiques, ...). Nous retiendrons :

a) les prises d'eau par des puits de captage côtiers,

b) les prises d'eau par infiltration sous les plages ou sous le fond marin,

c) les prises d'eau directes en mer ouverte, en surface ou au fond.

Malgré une transition continue, les notions de puits côtier et maritimes sont distinctes :

Les premiers sont suffisamment loin de la mer pour être mis en œuvre sans travaux maritimes et dépendre des propriétés hydrogéologiques du sol à l'échelle du site.

Les seconds, situés sur la plage ou dans les petits fonds, nécessitent des travaux maritimes et ne dépendent que localement des propriétés géotechniques du sol.

Le choix d'une solution doit être analysé à la lumière de nombreux critères : 
a) Critères physiques d'alimentation (importance et permanence du débit d'eau, souplesse d'adaptation du débit de la prise d'eau à celui demandé par l'usine de dessalement, distance entre la prise d'eau et l'usine de dessalement, ...).

b) Critères de qualité de l'eau brute obtenue (constance des propriétés physiques : température, salinité, masse volumique, ...), qualité mécanique (absence de végétaux, d'animaux, de larves, d'œufs, de zooplancton, de phytoplancton, de particules minérales ou de débris organiques en suspension, ...), qualité physico-chimique et biologique (absence de graisses, d'hydrocarbures, de colloïdes, de substances chimiques dissoutes ou en suspension, métaux lourds, micro-organismes, turbidité, ...), importance des prétraitements nécessaires en fonction du procédé de dessalement retenu.

c) Caractéristiques physiques offertes par le site du projet : géographiques, hydrogéologiques, géotechniques, hydrodynamiques, énergétiques, accessibilité, etc.

d) Critères environnementaux : accès à la côte, transit sédimentaire, caractéristiques géologiques du sol, salinité et température du concentrât, impacts sur le milieu humain et sur le milieu naturel terrestre et marin (physique, faune, flore, paysage, ...), possibilités administratives d'accès à la côte (zones protégées, autorisations, ...).

e) Critères économiques : coûts d'investissement, coûts d'entretien et de maintenance, coûts de filtration et de prétraitements de l'eau brute.

\section{Captage par puits côtiers}

Les puits de captage côtiers sont semblables aux puits de captage terrestres dans les nappes phréatiques, mais sont forés sur la frange littorale à des profondeurs suffisantes pour permettre une venue d'eau de mer ou saumâtre par infiltrations à travers le sol (voir figure 1a).
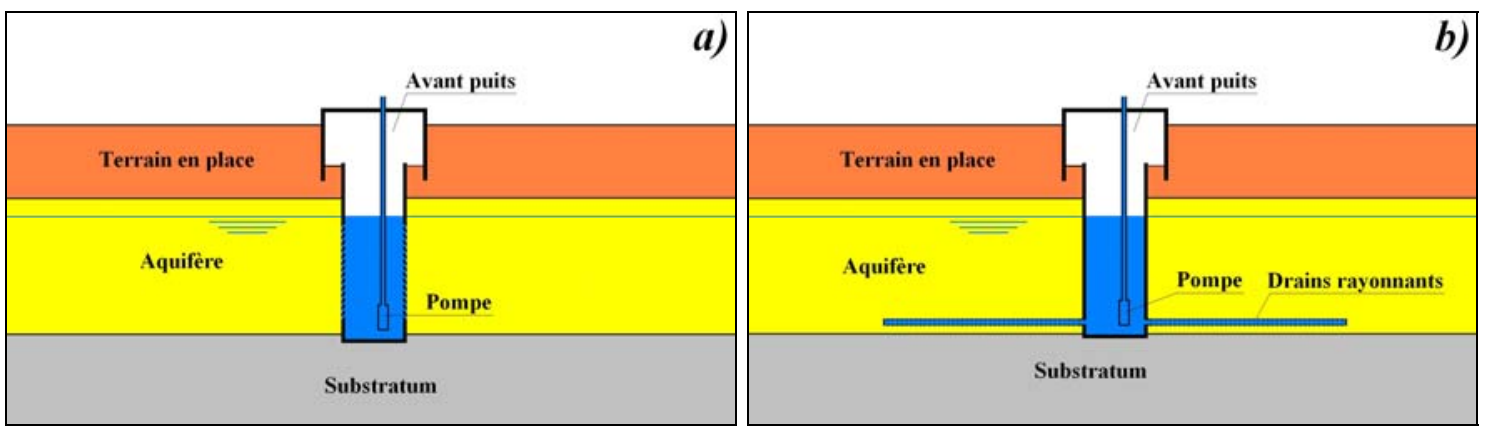

Figure 1. a) Puits côtier - b) Puits de plage à drains rayonnants (Ranney).

Ce type de captage présente les avantages suivants :

a) Il fournit une eau très bien adaptée au dessalement par osmose inverse : pas de matières en suspension ni d'algues, faible indice de colmatage (IC 2), faible concentration en matières organiques (COT<1 mg/l).

b) Il permet de limiter et de simplifier les prétraitements. 
La connaissance de la Mer :

un vecteur du développement durable en Méditerranée

c) Il peut être utilisé dans les zones côtières inhospitalières constituées de falaises ou de platiers rocheux battus par une agitation importante et permanente.

d) Il est insensible au transit sédimentaire.

Il présente toutefois des inconvénients :

a) Il ne fournit que de faibles débits (risques de colmatage du sol et de dénoyage du puits si la vitesse de filtration est trop importante), de l'ordre de 10 à 50 l/s, par puits qui doivent être séparés de plusieurs centaines de mètres pour éviter les interférences entre leurs rabattements de la nappe salée.

b) Possibilité d'interactions entre la nappe salée et la nappe d'eau douce qui coule vers la mer (constance de la salinité nécessaire au bon rendement de l'installation).

c) Nécessité d'investigations géologiques et hydrogéologiques poussées.

d) Nécessité du suivi des caractéristiques physiques, chimiques et biologiques de l'eau.

\section{Captage par infiltration sous les plages ou sous le fond marin}

L'eau de mer peut être captée sous une plage ou une avant plage par des puits maritimes, des tranchées drainantes, des drains foncés ou forés ou des champs drainants.

\subsection{Puits maritimes}

Les puits maritimes sont semblables à des puits côtiers, mais ils sont directement forés dans l'aquifère d'eau de mer ou dans le biseau salé, en haut de plage, sur la plage, sur le bas estran ou dans les petits fonds. Leur drainage peut être amélioré avec des drains horizontaux rayonnants dont la longueur est généralement de l'ordre de quelques diamètres (5 à 20) du puits (puits Ranney) (voir figure 1b). Ce type de captage présente les avantages suivants :

a) Il fournit une eau de très bonne qualité qui peut être comparable à celle des puits côtiers selon l'épaisseur des terrains traversés et la vitesse de filtration.

b) Il fournit des débits plus importants que les puits côtiers (plus grande perméabilité du sol), de l'ordre de 50 à 300 l/s, par puits qui doivent être séparés de 100 à 150 m pour éviter les interférences entre leurs rabattements de la nappe salée.

c) Pas d'interaction entre la nappe salée et la nappe d'eau douce.

d) Les puits maritimes nécessitent une profondeur moindre que les puits côtiers.

Il présente toutefois des inconvénients :

a) Il nécessite un accès à la plage pour la construction et l'exploitation (travaux maritimes), ce qui rend difficile son utilisation sur les côtes à falaises.

b) Il peut devenir sensible au transit sédimentaire si les vitesses de filtration sont suffisantes pour engendrer un engraissement significatif de la plage.

c) Il nécessite des investigations géologiques et hydrogéologiques poussées 


\subsection{Tranchées drainantes}

Le captage se fait par une conduite perforée ensouillée au-dessous du niveau des plus basses mers, dans une tranchée au milieu d'un lit de graviers ou de ballast entouré d'un filtre géotextile. La conduite perforée est reliée par une conduite étanche à un puits qui se remplit d'eau de mer sous l'effet de la gravité. Le drain peut être protégé contre les affouillements (zone de déferlement) en l'enfouissant plus profondément. Les graviers sont alors recouverts d'une couche de sable filtrant et d'une protection perméable de type "rip-rap".

a) Tranchée parallèle au trait de côte sous la plage (voir figure 2a) : L'eau de mer s’infiltre dans le drain par le côté et partiellement par-dessus et par-dessous. La section du lit de graviers peut être plus haute que large.

b) Tranchée normale au trait de côte sous l'avant plage (voir figure 2b) : L'eau de mer s'infiltre dans le drain essentiellement par le dessus. La tranchée est donc recouverte, soit avec les matériaux d'origine si leurs qualités filtrantes sont satisfaisantes, soit avec des matériaux d'apport de plus grande perméabilité.

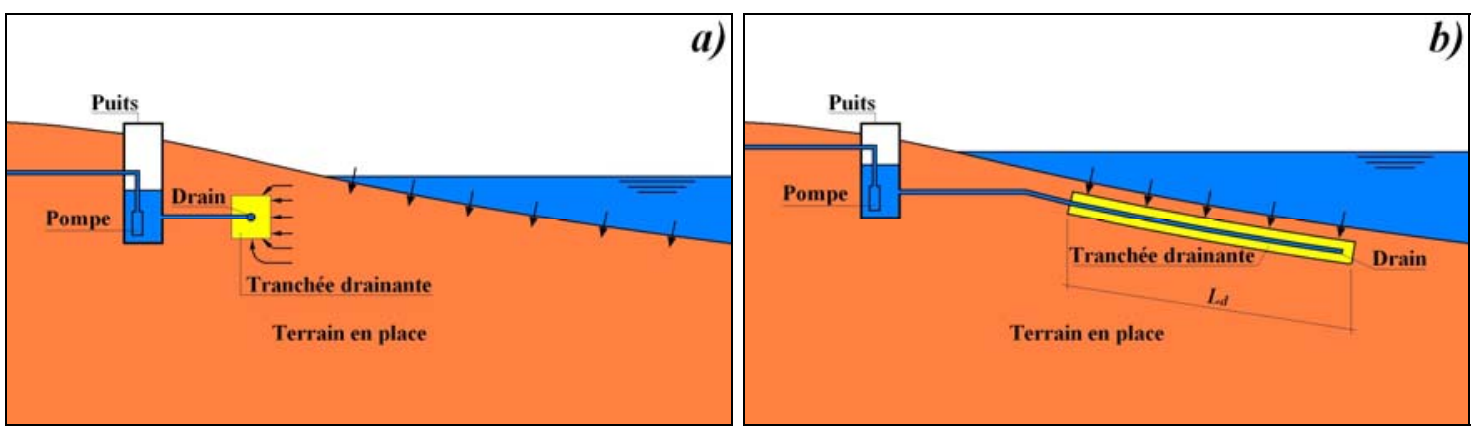

Figure 2. Tranchée drainante a) parallèle à la côte - b) normale à la côte.

\subsection{Drains foncés ou forés subhorizontaux}

Une variante de la tranchée normale au trait de côte consiste à foncer (terrains meubles) ou à forer (terrains durs fracturés) un drain sous la mer. La longueur du drain permet d'ajuster le débit en fonction des caractéristiques du sol. Cette technique, de type forage dirigé, permet d'aller loin sous la mer sans altérer l’intégrité des fonds marins.

\subsection{Champs drainants}

L'installation de plusieurs drains, parallèles ou convergents, en tranchées drainantes ou par forages dirigés, permet de limiter l'éloignement vers le large. En filtration semilente $(0.2$ à $0.6 \mathrm{~m} / \mathrm{h})$, un champ drainant peut fournir un débit surfacique de 0.06 à $0.18 \mathrm{l} / \mathrm{s} / \mathrm{m}^{2}$. La première usine de dessalement alimentée par un champ drainant est celle de Fukuoka au Japon - 2005 : 2 ha pour $1.2 \mathrm{~m}^{3} / \mathrm{s}$ d'eau de mer ; soit $0.06 \mathrm{l} / \mathrm{s} / \mathrm{m}^{2}$ (PANKRATZ, 2008). 
La connaissance de la Mer :

un vecteur du développement durable en Méditerranée

\section{Prises d'eau de mer directes}

\subsection{Prise d'eau par canal d'amenée}

Plus le débit de la prise d'eau de mer est important et moins les pertes de charge sont acceptables. Une prise d'eau par un canal à la côte s'impose donc pour les très gros débits. Les centrales électronucléaires ont été conçues sur ce schéma ( 45 m³/tranche).

\subsection{Prise d'eau par des puits immergés}

La filtration en bassin consiste à capter l'eau à travers une grille grossière (10 à $20 \mathrm{~cm}$ ) pour retenir les corps solides pouvant obstruer ou endommager la conduite. Il n'est pas possible d'installer une pompe sur la conduite et l'eau doit être amenée par un siphon (gravitaire ou à dépression) dans un bassin de pompage où elle est filtrée. Ce procédé est généralement adopté pour les gros débits (quelques $\mathrm{m}^{3} / \mathrm{s}$ à quelques dizaines de $\mathrm{m}^{3} / \mathrm{s}$ ), par exemple pour le refroidissement des centrales électriques moyennes.

a) Prise d'eau en surface par des conduites : La conduite est supportée par un ouvrage (passerelle dédiée (wharf), corps de digue ou de jetée, épi, ...). Munie d’un dispositif d'amorçage sous vide, elle plonge sous la surface libre et aspire l'eau à travers une tulipe conçue pour éviter les vortex et, dans le cas de prises peu immergées, la dépression de la surface libre. (voir figure 3a). La tulipe est protégée par des grilles.

b) Prise d'eau profonde par des galeries ou des conduites : L'eau est amenée au moyen d'une galerie forée ou d'une conduite ensouillée dans la zone de déferlement, puis posée au fond et lestée ou ancrée au-delà. L'eau est aspirée à travers une tulipe ou un puits muni de grilles (voir figure $3 b$ ).

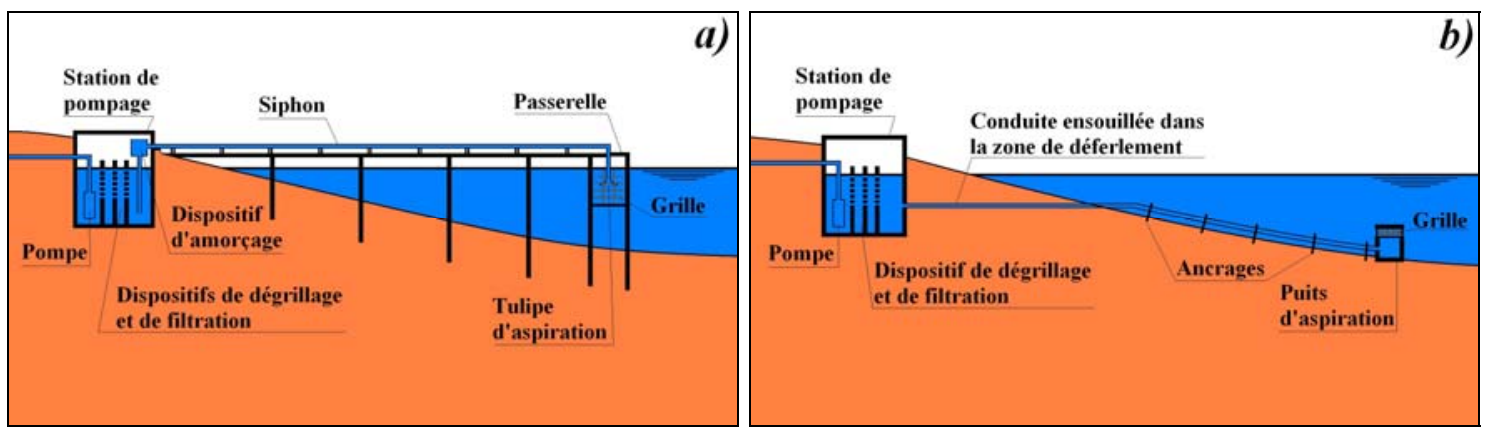

Figure 3. Prise d'eau a) en surface - b) au fond.

\subsection{Prise d'eau à crépines}

La filtration à la prise consiste à prendre l'eau à travers une crépine dont le diamètre des mailles va de quelques dizaines de $\mu \mathrm{m}$ à quelques $\mathrm{mm}$. Cette filtration permet l'installation d'une pompe directement sur la conduite d'aspiration sans l'exposer (détérioration par les corps solides et les poissons, abrasion par les particules fines, ...). Ce procédé est généralement réservé aux faibles débits et aux débits moyens (de quelques 
$\mathrm{m}^{3} / \mathrm{h}$ à quelques centaines de $\mathrm{m}^{3} / \mathrm{h}$ ). Un nettoyage périodique de la crépine est nécessaire (soufflage d'eau ou d'air comprimé, intervention en mer).

a) Prise d'eau à crépine en surface : Le principe est le même que précédemment, mais la tulipe est remplacée par une crépine, verticale ou horizontale, installée à proximité de la surface libre. La pompe est située sur la conduite, soit à terre, soit l'extrémité de la passerelle pour refouler l'eau sur la presque totalité de la longueur de la conduite ; l'électricité doit alors être amenée sur la passerelle. L’utilisation de pompes immergées complique la maintenance et l'entretien pour des installations difficilement accessibles.

b) Prise d'eau à crépine au fond : Le principe est le même que précédemment, mais l'eau est aspirée à travers une crépine installée au-dessus du fond et protégée par une bâche ou par une grille. La station de pompage est située à terre à une altitude voisine du niveau des plus basses mers. L’eau est aspirée dans la conduite et refoulée ensuite vers des installations situées à des niveaux plus élevés. Pour des pertes de charge pas trop importantes, la venue d'eau peut être gravitaire jusqu'à la station de pompage.

\section{Rejet à la mer du concentrât}

A la sortie de l'usine de dessalement, le flux d'eau de mer brute a été séparé en deux parties : un flux d'eau douce et un flux de saumure qui contient l'ensemble du sel.

Il existe trois procédés pour se rejeter le concentrât à la mer :

a) le rejet à la côte au moyen d'un canal,

b) le rejet au large au moyen d'un émissaire,

c) le rejet par infiltration sous la mer.

Le choix du type de rejet dépend surtout de critères environnementaux et économiques.

\subsection{Rejet à la côte par surverse ou par un canal}

Le rejet à la côte consiste soit à laisser tomber le concentrât dans la mer depuis une falaise, soit à le laisser couler sur la plage, sur un seuil en béton ou en enrochements, ou encore, à le rejeter dans un canal qui se jette à la mer comme un cours d'eau (voir figure 4a). Cette solution, analogue à la prise d'eau par un canal, consiste simplement à organiser et régulariser le rejet.

Le canal peut être un ouvrage de génie civil ou bien un ouvrage en enrochements ("riprap"). Une grille de protection évite les risques d'encombrement par des corps étrangers flottants. Ce type de rejet, très polluant, a été retenu pour l'usine d'Ashkelon en Israël avec un débit de rejet de l'ordre de $4 \mathrm{~m}^{3} / \mathrm{s}$. 
La connaissance de la Mer :

un vecteur du développement durable en Méditerranée

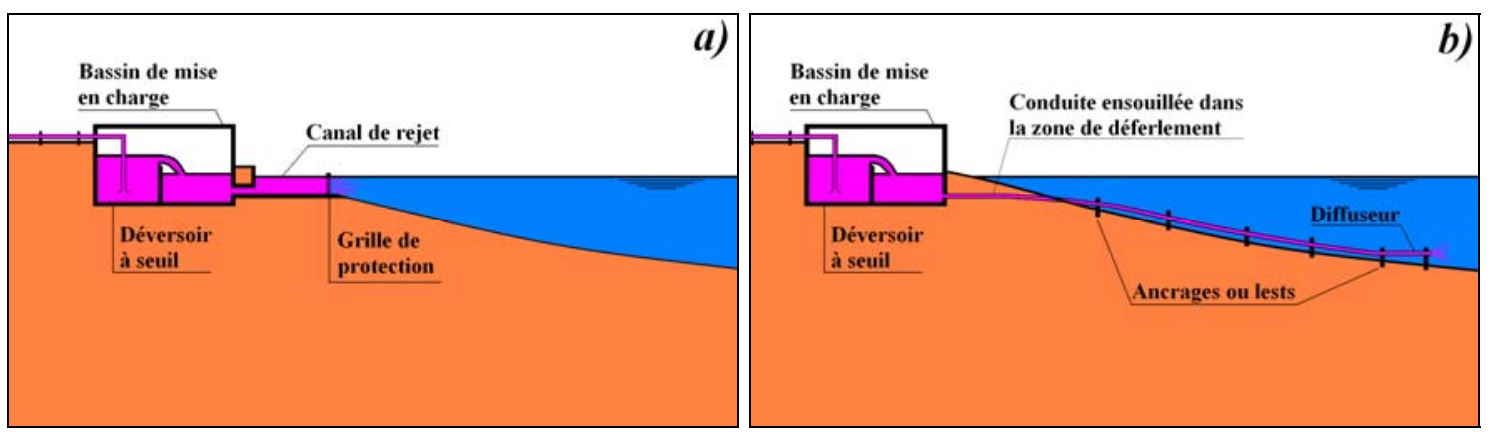

Figure 4. Rejet d'effluent a) en canal - b) par un émissaire.

\subsection{Rejet au large par une galerie ou un émissaire}

La saumure est rejetée à la mer par une galerie forée (sols durs) ou foncée (sols meubles) sous la mer ou par un émissaire généralement ensouillé dans la zone de déferlement puis posé au fond de la mer (voir figure 4b). Son extrémité est munie d'un diffuseur plus ou moins sophistiqué selon l'impact toléré sur la faune et la flore. Il est ancré ou lesté pour rester stable sous l'action de la houle et des courants marins. Le dispositif de fixation de la conduite dépend de son poids, selon qu'elle lourde (fondrière) ou légère, et de la nature du sol qui peut être meuble ou résistant. Les émissaires légers ensouillés doivent être lestés pour éviter qu'ils ne remontent à la surface du sol sous l'action des pressions alternées dans le sol.

\subsection{Rejet par infiltration sous les plages}

Le rejet de la saumure par infiltration sous la mer est le pendant de la prise d'eau par infiltration sous les fonds marins. Cette solution ne semble pas répandue (usine test de Long Beach en Californie - 2005). L’intérêt de ce procédé n'est pas évident et l’opportunité de l'utiliser doit être analysée avec circonspection.

\subsection{Station de refoulement du concentrât}

Sauf si la topographie ne le permet pas, la station de refoulement de la saumure doit être gravitaire. La station de refoulement comprend une chambre de rejet de l'effluent suivie d'une chambre de mise en charge permettant l'entrée dans la conduite de rejet (voir figure 4). Les deux chambres sont séparées par un seuil, ce qui permet :

a) de se passer des pompes de refoulement et des organes de protection afférents,

b) d'éliminer les influences de l'écoulement aval sur l'écoulement amont,

c) de maintenir à l'amont une pression indépendante du niveau de la mer et quasi indépendante du débit.

\subsection{Diffuseur en sortie d'émissaire}

L'écart de densité entre la saumure et l'eau de mer favorise l'établissement d'un courant de densité qui s'écoule lentement sur le fond marin. La dilution de la masse d'eau hy- 


\section{A best knowledge of the Sea: \\ A sustainable development vector in Mediterranean}

persaline dans le milieu ambiant se fait lentement et nécessite des distances très grandes pouvant atteindre plus de $4 \mathrm{~km}$, comme à Alicante en Espagne (FERNANDEZTORQUEMADA, 2005). La forme du tronçon de sortie influence la propension de l'effluent à se diluer dans la mer. Un diffuseur optimisé possède de nombreux orifices répartis sur une distance de plusieurs dizaines de mètres, voire plus. Les exutoires sont surélevés au-dessus du fond d'un à deux mètres. Chaque exutoire a une section limitée et son axe est incliné de $45^{\circ}$ vers le haut ; l'impact du jet sur le fond augmente la dilution. Toutefois, la présence d'un diffuseur augmente les pertes de charge et empêche d'utiliser un obus pour nettoyer la conduite.

\section{Contraintes environnementales}

Du fait de sa salinité et de sa concentration en substances chimiques (prétraitement, rinçage des membranes et produits chimiques utilisés contre les salissures, l'entartrage et la corrosion) (LATTERMANN \& HÖPNER, 2003), l'écoulement engendre des impacts sur la faune et la flore, notamment sur les organismes benthiques sténohalins :

a) La disparition de toute vie à l'extrémité de l'émissaire sur une aire pouvant atteindre un hectare.

b) L'anoxie des fonds marins engendrée par les espèces benthiques, consommatrices d'oxygène, lors des périodes de faible hydrodynamisme.

c) La carence en lumière des fonds marins affecte la photosynthèse des espèces marines végétales ; phénomène accentué par la turbulence due au jet.

d) Des réductions massives de populations d'oursins, d'étoiles de mer, de crevettes, etc.

e) Une augmentation de la mortalité des herbiers de posidonies avec des tissus nécrosés et d'abondantes chutes de feuilles.

f) etc.

Les seuils d'affection varient considérablement d'une espèce à l'autre.

\section{Tenue à la mer des ouvrages maritimes}

Les ouvrages de prise d'eau directe et de rejet sont composés d'éléments : conduites, ancrages, lests, bétonnage, ensouillage, puits d'aspiration, diffuseur. Soumis à l'action des courants et de la houle, ils subissent des efforts, parfois très importants, qu'il faut évaluer pour dimensionner l'ouvrage en assurant sa stabilité externe (glissement, basculement, arrachement), mais aussi sa stabilité interne (déformation, endommagements structurels, rupture).

Compte tenu des rapports entre les dimensions caractéristiques des divers éléments et les longueurs d'onde des houles à prendre en considération, la mise en œuvre de logiciels de calculs de diffraction - radiations reste exceptionnelle. Les efforts sont évalués avec la formule asymptotique de Morison généralisée qui distingue :

a) l'effort d'inertie, proportionnel au volume immergé et à l'accélération du fluide,

b) l'effort de traînée, proportionnel au maître couple et au carré de la vitesse du fluide, 
La connaissance de la Mer :

un vecteur du développement durable en Méditerranée

c) l'effort de portance, proportionnel à une surface et au carré de la vitesse du fluide.

Les trois coefficients de proportionnalité sont des coefficients hydrodynamiques empiriques $\left(C_{M}, C_{D}\right.$ et $\left.C_{L}\right)$, issus de la littérature. Ils ont été déterminés par des essais sur des modèles physiques ou numériques (Navier-Stokes), sous des hypothèses précises.

Les coefficients hydrodynamiques pour des conduites plongées dans un fluide illimité et soumises à un courant permanent et uniforme sont connus depuis longtemps. Les études des écoulements oscillants ou orbitaux, en particulier à proximité du fond ou de la surface libre, sont relativement récentes et n’ont été que partiellement et insuffisamment diffusées dans les domaines maritimes non liés à l'exploitation pétrolière en mer. Une grande attention doit donc être portée aux coefficients hydrodynamiques utilisés dans les formules de Morison qui dépendent naturellement du nombre de Reynolds, de la rugosité relative de la conduite et du nombre de Strouhal, mais aussi du nombre de Keulegan et Carpenter $K_{c}$ et de la distance relative $E_{D}$ de la conduite au fond (SUMER \& FREDSØE, 2006).

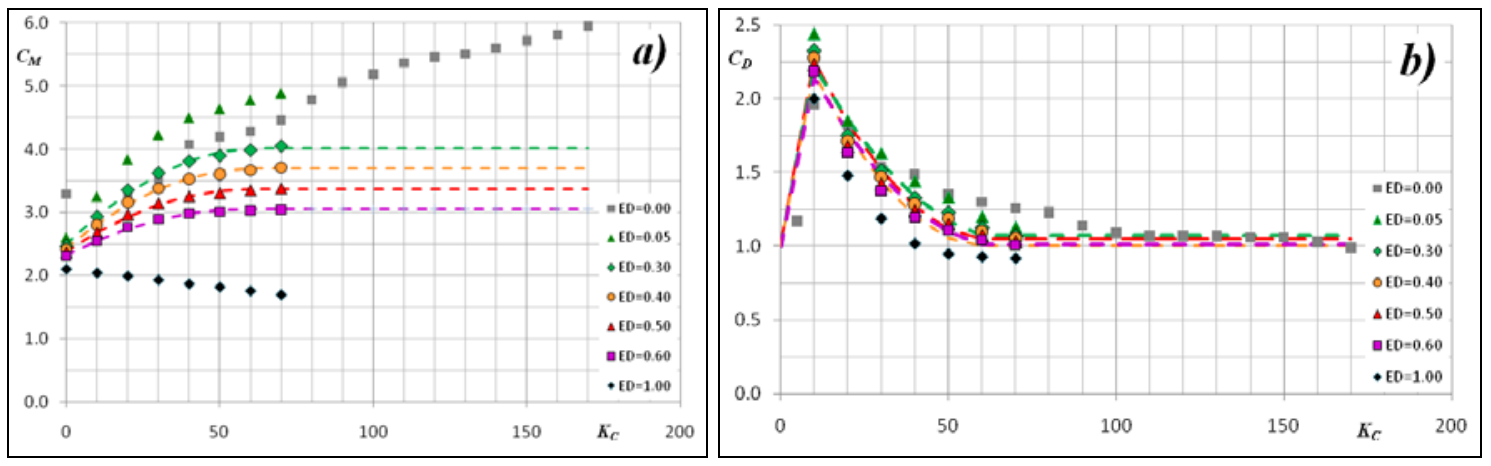

Figure 5. Coefficients hydrodynamiques a) de masse ajoutée - b) de traînée.

La proximité du fond augmente considérablement les efforts, surtout pour les petits nombres de Keulegan et Carpenter (amplitudes des oscillations pas trop grandes devant le diamètre : de l'ordre de moins de 50 à 100 fois) ; ce qui est couramment le cas pour les conduites des prises d'eau et les émissaires. Les importantes variations des coefficients avec la période imposent de recourir à l'analyse spectrale (voir figures 5).

\section{Critères de choix du site d'implantation}

Lors du choix d'un site d'implantation d'une usine de dessalement, des contradictions peuvent exister entre les critères dédiés à la prise d'eau et ceux dédiés au rejet :

a) Pour la prise d'eau, on recherchera une zone éloignée de tout rejet, protégée contre l'agitation et permettant d'assurer la pérennité de l'alimentation quelles que soient les conditions météorologiques et océaniques. 
b) Pour le rejet, on privilégiera un site ouvert, présentant un fort hydrodynamisme (coutants, agitation) avec la possibilité de diluer la saumure avec d'autres rejets présentant une complémentarité (moindre température, moindre salinité, etc.).

La disposition relative de la prise et du rejet est également très importante pour limiter les risques de recyclage direct, indirect ou différé des rejets vers la prise d'eau.

Les facteurs les plus importants sont :

a) La topographie du rivage et l'altitude du site qui conditionnent la hauteur de pompage des eaux d'alimentation et la mise en charge d'un émissaire gravitaire.

b) La couverture sédimentaire et le transit sédimentaire qui conditionnent la profondeur de la prise d'eau et/ou du rejet ainsi que le besoin de filtration des eaux prélevées.

c) La bathymétrie des fonds qui conditionne la longueur de la conduite de la prise d'eau et/ou de l'émissaire.

d) La vulnérabilité de la zone de rejet et les conditions requises pour une bonne diffusion de l'effluent qui conditionnent le facteur de conversion ou la nécessité d'une dilution avant rejet.

\section{Critères de choix du procédé de prise d'eau}

Les différents procédés de prise d'eau sont analysés en fonction du débit demandé, du procédé de dessalement retenu et des caractéristiques du site ; les prises indirectes étant privilégiées pour la qualité de leurs eaux.

Un compromis doit ensuite être trouvé entre les critères techniques (qualité de l'eau, topographie, etc.), économiques (investissement et fonctionnement) et environnementaux.

Pour des débits d'eau de mer inférieurs à 0.5 m³/s, les coûts d'investissements sont généralement à l'avantage des procédés de prises indirectes (voir figure 6). Au-delà les procédés de prises directes deviennent moins coûteux. Le coût des ouvrages de prise d'eau de mer et de rejet des saumures représente couramment 5 à $15 \%$ de l'investissement global, voire plus pour les unités de faible capacité. Les eaux des prises indirectes nécessitant moins de prétraitements, induisent des coûts de fonctionnement de 10 à $20 \%$ plus faibles.

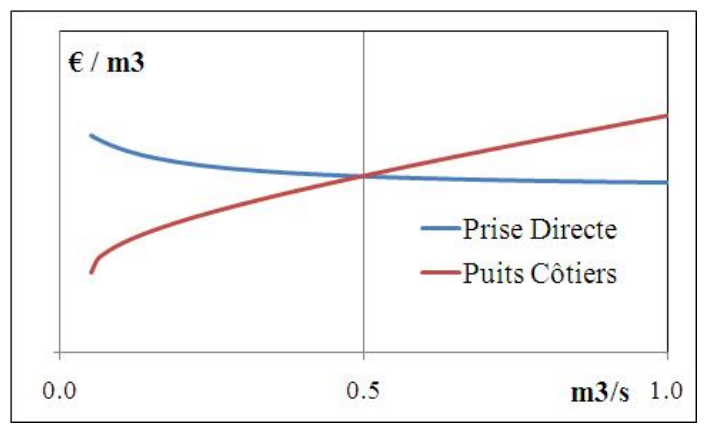

Figure 6. Coûts comparatifs des prises d'eau directes et indirectes selon le débit. 
La connaissance de la Mer :

un vecteur du développement durable en Méditerranée

\section{Remerciement}

Une partie de ce travail de synthèse a été préparée dans le cadre de l'étude stratégique sur le dessalement de l'eau de mer au Maroc (BOUGIS, 2009) réalisée, en collaboration avec la Société ADI du Groupe ONA (Rabat), pour le Secrétariat d'Etat chargé de l'Eau et de l'Environnement auprès du Ministère de l'Energie, des Mines, de l'Eau et de l'Environnement du Maroc. Que tous ceux qui ont rendu ce travail possible en soient remerciés.

\section{Références bibliographiques}

BOUGIS J. (2009). Contribution pour les aspects hydrodynamiques à l'étude stratégique sur le dessalement de l'eau de mer au Maroc. Rapport RET.08.478.02.01, 48 p. FERNANDEZ-TORQUEMADA Y. (2005). Preliminary results of the monitoring of the brine discharge produced by the SWRO desalination plant of Alicante (SE Spain), Desalination, $\mathrm{n}^{\circ}$ 182, pp 395-402.

LATTERMANN S., HÖPNER T. (2003). Seawater desalination. Impacts of Brine and Chemical Discharge on the Marine Environment, L'Aquila: Desalination Publication, $142 \mathrm{p}$.

MAUREL A. (2001). Dessalement de l'eau de mer et des eaux saumâtres. Technique \& Documentation, Paris, 226 p.

PANKRATZ T. (2008). Global overview of seawater desalination intake issues. Alden Desalination Intake Solutions Workshop, Holden (Massachusetts), 16 October, 46 p. [URL http://www.aldenlab.com ]

SUMER B.M., FREDSØE J. (2006). Hydrodynamics around cylindrical structures. Advanced Series on Ocean Engineering, World Scientific Publishing Co, Singapore, Vol. 26, 530 p. 